\title{
Pseudomesotheliomatous carcinoma of the lung in the parietal pleura
}

\author{
Ae Ri An, Kyoung Min Kim, Jong Hun Kim, Gong Yong Jin, Young Hoon Choe, Myoung Ja Chung \\ Department of Pathology, Jeonbuk National University Medical School, Jeonju, Korea
}

Pseudomesotheliomatous carcinoma of the lung (PCL) is a rare and unique subtype of lung adenocarcinoma located in the pleura, which clinically and/or radiologically mimics the growth of malignant mesotheliomas (MM). It is characterized by pleural thickening without lung mass; however, ultrastructural, immunohistochemical, and molecular characteristics suggest type II pneumocyte origin [1]. The original definition of this cancer by Harwood et al. described it as a unique form of lung cancer [1]. PCL is known to have a very poor prognosis due to diffuse pleural involvement, multiple extrapulmonary metastasis, and no effective treatment [2]. Advances in our understanding of the genomic alterations of cancers have profoundly altered the treatment options and prognosis for cancers. However, few studies are available on the molecular analysis of PCLs regarding genetic alteration. In this study, we report a case of PCL in the parietal pleura with results on the genetic alteration of cancer-related genes using a targeted next-generation sequencing (NGS) method.

\section{CASE REPORT}

A 69-year-old male patient presented with dyspnea and recurrent pleural effusion. He was a current smoker with a 30-pack year. He had no history of chronic pulmonary disease or asbestos exposure. The patient received thoracentesis and antibiotic treatment, but his condition did not improve. A computed tomography $(\mathrm{CT})$ of the chest revealed left pleural effusion and pleural thickening with multiple nodules, suggestive of metastatic carcinoma. No parenchymal mass was observed in the lung (Fig.

Received: September 27, 2019 Revised: November 11, 2019

Accepted: November 14, 2019

Corresponding Author: Myoung Ja Chung, MD, PhD

Department of Pathology, Jeonbuk National University Medical School, 567

Baekje-daero, Deokjin-gu, Jeonju 54896, Korea

Tel: +82-63-270-3072, Fax: +82-63-270-3135, E-mail: mjchung@jbnu.ac.kr
1A). Positron emission tomography (PET)-CT showed fluorodeoxyglucose uptake in the areas of pleural thickening (Fig. 1B). Video-assisted thoracoscopic surgery (VATS) showed diffuse multiple nodules in the parietal pleura, and a biopsy was performed for histologic diagnosis. The histopathological examination presented glandular and cord-like structures of tumor cells within desmoplastic stroma (Fig. 2A). Under high-power field, a hobnail-like appearance of tumor cells with hyperchromatic nuclei and inconspicuous nucleoli was identified (Fig. 2B). Immunohistochemistry (IHC) was performed using an automatic immunostainer (BenchMark XT, Ventana Medical Systems, Tucson, AZ, USA) according to the manufacturer's instructions. Immunohistochemically, the tumor cells were positive for thyroid transcription factor-1 (ready to use [RTU], Ventana Medical Systems) (Fig. 2C), carcinoembryonic antigen (RTU, Ventana Medical Systems), Napsin A (RTU, Ventana Medical Systems), and epithelial membrane antigen (1:50, Dako, Glostrup, Denmark). Calretinin (RTU, Ventana Medical Systems) (Fig. 2D), cytokeratin 5/6 (RTU, Ventana Medical Systems), Wilms' tumor product-1 (1:100, Dako), and D2-40 (RTU, Ventana Medical Systems) were negative. With these immunohistochemical results and clinical presentation, the tumor was diagnosed as PCL. The patient refused chemotherapy and is only receiving treatment for pleural effusion.

Targeted NGS was performed using formalin-fixed paraffinembedded (FFPE) tumor tissue. Total nucleic acid was isolated from FFPE tumor tissue using RecoverAll Total Nucleic Acid Isolation Kit for FFPE according to the manufacturer's specifications (Thermo Fisher Scientific, Waltham, MA, USA). The samples were sequenced using the Oncomine Comprehensive Assay Cancer Panel (Ion torrent S5 XL, Thermo Fisher Scientific), which covers 2737 amplicons (2530 DNA + 207 RNA) within 143 cancer-related genes. The number of mapped DNA reads was 8,755,214 ( $\geq \mathrm{Q} 20)$ and the mean coverage per target ampli- 

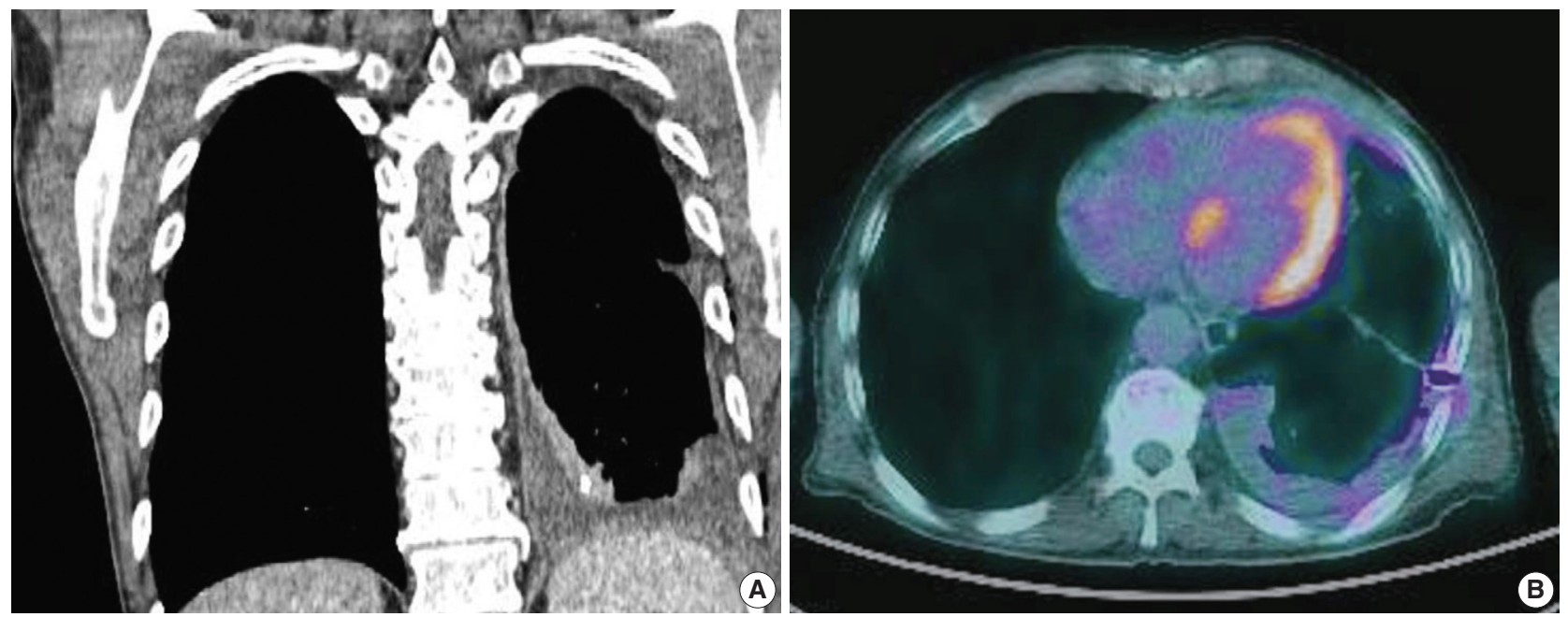

Fig. 1. (A) Computed tomography (CT) of the chest shows the extremely thickened pleura along the left lobe. (B) Positron emission tomography-CT shows fluorodeoxyglucose uptake in the left pleural thickening areas.
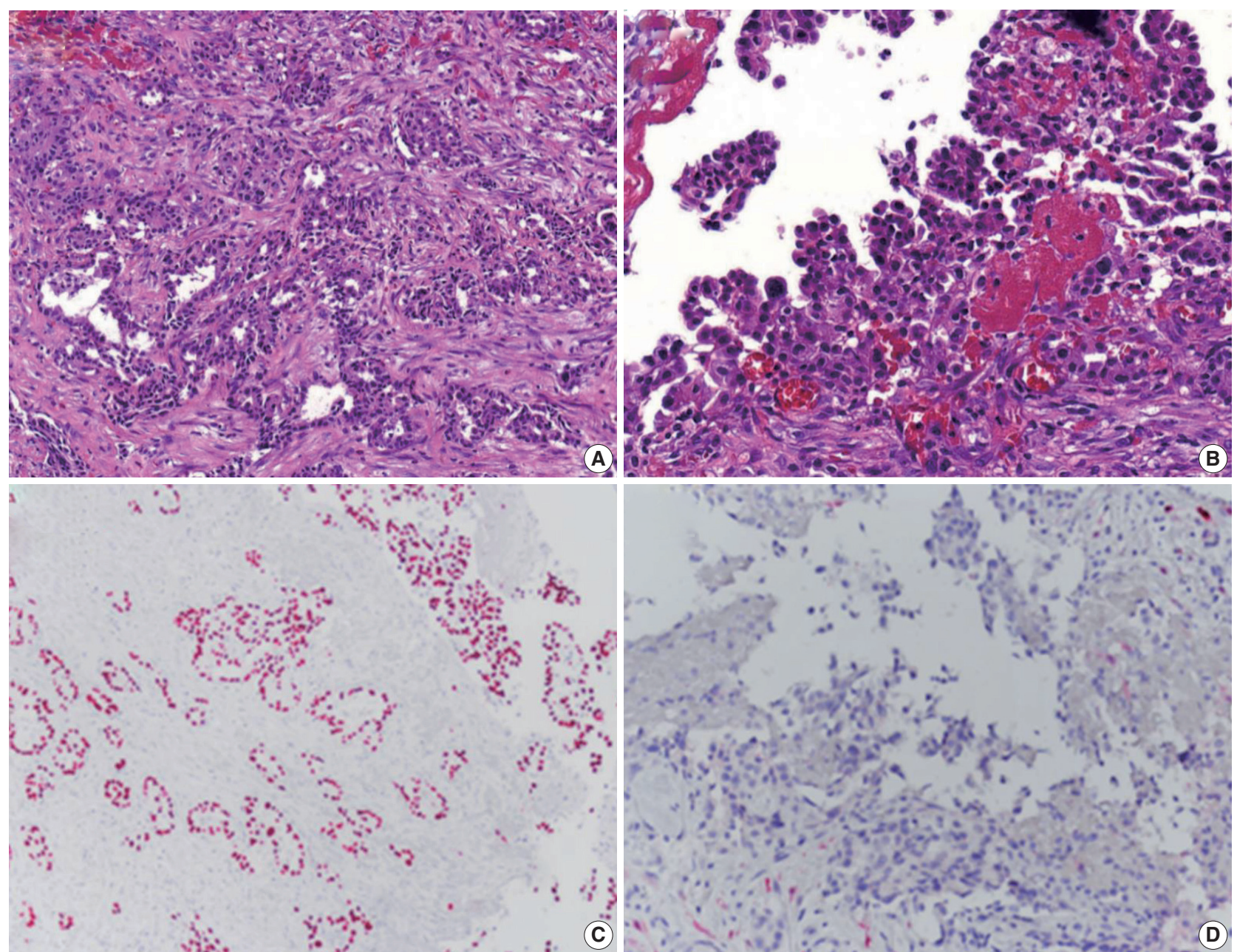

Fig. 2. Histopathological sections showing tumor cells with glandular and papillary, cord-like structures (A) and a hobnail-like appearance of tumor cells with irregular and hyperchromatic nuclei resembling malignant mesothelioma (B). Immunohistochemical evaluation demonstrates the tumor cells to be positive for thyroid transcription factor-1 (C) and negative for calretinin (D). 
con was $\times 3,616$. The percentage of amplicons that were covered by greater than $20 \%$ of the mean amplicon coverage was $95 \%$. Reads were aligned to the hg19 reference genome and allele frequencies $<5 \%$ were excluded. We identified one pathogenic somatic mutations in splicing factor 3B subunit 1 (SF3B1) gene, NM_012433.3(SF3B1):c.2098A > G (p.Lys700Glu).

\section{Ethics statement}

This study was approved by the Institutional Review Board of Chonbuk National University Hospital with a waiver of informed consent (IRB No. 2019-04-040).

\section{DISCUSSION}

PCLs were first reported by Harwood et al. in 1976 [1]. The authors defined this tumor entity as a malignant epithelial neoplasm, which presents with radiological, macroscopic, and microscopic features similar to those of diffuse malignant pleural mesothelioma [1]. PCL is mostly found in men, and the age of onset is between the sixth and seventh decades of life. The majority of patients are cigarette smokers [3]. In association with asbestos exposure, some researchers reported on the presence of asbestos bodies in cancer tissue, and Koss et al. [3] reported the possibility of occupational exposure in $21 \%$ of the patients. However, the relationship between cancer development and asbestos exposure is unclear. The tumor growth pattern makes it difficult to differentiate PCL from MM based on radiologic findings alone, and histologic confirmation is needed. IHC provides an adequate sensitivity and specificity for distinguishing PCL from MM. The tumor cells are positive for thyroid transcription factor-1, Napsin A, epithelial membrane antigen, and carcinoembryonic antigen and negative for mesothelial markers such as calretinin and D2-40.

PCL is characterized mainly by visceral pleural thickening; however, our case showed a unique presentation of only parietal pleural thickening without visceral pleural thickening. These clinical features created a diagnostic difficulty, and in this case, it was important to recognize PCL for an accurate diagnosis.

PCL shows a distinctly different biological behavior from the usual type of adenocarcinoma of the lung. Because of its extensive invasion of the pleura and lack of effective treatment, patients have a poor prognosis [4]. Recently, identification of the genetic alteration of cancer-related genes through sequencing in cancer has been widely used for application of targeted cancer treatment. Because PCL does not yet have an established guideline on effective treatment and the prognosis is poor, it would be meaningful to know the genetic alterations of PLC in terms of therapeutic development. No studies about genetic alterations in PCL have been reported [5]. We investigated the mutational status of cancer-related genes using targeted NGS and identified genetic alteration in $S F 3 B 1$ gene, which is involved in transcription and mRNA processing. The mutation of SF3B1 gene has been recurrently observed in hematologic malignancies, breast cancer, pancreas cancer, prostate cancer, and others. SF3B1 mutations have been reported to be associated with poor outcome and drug resistance in chronic lymphocytic leukemia [6]. Although neither the mutation of known genes that have target drugs nor driver mutation were observed in this study, an accumulation of information on genetic alteration is expected to be useful for understanding the pathogenesis or developing an effective treatment in the future.

The histogenesis of PCL is not clear. Harwood et al. [1] reported a small subpleural adenocarcinoma associated with some PCL and suggested a possible origin of PCL, as this small subpleural tumor became widely disseminated via subpleural lymphatics. A second suggestion is that the fibrous thickening of the pleura may be an early event. Furthermore, the cancer developed in subpleural lung parenchyma and subsequently spread rapidly through the thickened pleura. In the present case, VATS showed diffuse pleural thickening with multiple nodules in the parietal pleura instead of the visceral pleura. Neither chest CT nor PET-CT revealed lung parenchymal lesions. In our case, with these presentation characteristics, the possibility of different histogenesis can be considered. The possibility of pulmonary heterotopia of the pleura can be considered based on reports that lung tissue is found in an ectopic location [7]. However, there is no report of ectopic lung tissue in the parietal pleura, and there is no evidence to support this hypothesis in our case.

We report a case of PCL presented with parietal pleural thickening without lung parenchymal lesions. The present report is the first case of PCL with gene mutation results approached through NGS.

\section{ORCID}

Ae Ri An: https://orcid.org/0000-0002-6047-1627

Kyoung Min Kim: https://orcid.org/0000-0001-7074-7183

Jong Heon Kim: https://orcid.org/0000-0001-9289-1178

Gong Yong Jin: https://orcid.org/0000-0002-1426-554X

Young Hoon Choe: https://orcid.org/0000-0002-7982-7822

Myoung Ja Chung: https://orcid.org/0000-0003-4165-7167 


\section{Author Contributions}

Conceptualization: MJC.

Data curation: ARA, KMK, YHC, JHK, GYJ.

Investigation: ARA, KMK.

Writing: ARA, MJC.

\section{Conflicts of Interest}

The authors declare that they have no potential conflicts of interest.

\section{Funding}

No funding to declare.

\section{REFERENCES}

1. Harwood TR, Gracey DR, Yokoo H. Pseudomesotheliomatous carcinoma of the lung: a variant of peripheral lung cancer. Am J Clin Pathol 1976; 65: 159-67.

2. Attanoos RL, Gibbs AR. 'Pseudomesotheliomatous' carcinomas of the pleura: a 10-year analysis of cases from the Environmental Lung Disease Research Group, Cardiff. Histopathology 2003; 43: 444-52.

3. Koss MN, Fleming M, Przygodzki RM, Sherrod A, Travis W, Hochholzer L. Adenocarcinoma simulating mesothelioma: a clinicopathologic and immunohistochemical study of 29 cases. Ann Diagn Pathol 1998; 2: 93-102.

4. Vuković J, Plavec G, Aćimović S, et al. Pseudomesotheliomatous carcinoma of the lung. Vojnosanit Pregl 2016; 73: 1168-72.

5. Sakata S, Sakamoto Y, Takaki A, Ishizuka S, Saeki S, Fujii K. Reversible restrictive lung disease in pseudomesotheliomatous carcinoma in a lung harboring a HER2-mutation. Intern Med 2018; 57: 2223-6.

6. Rossi D, Bruscaggin A, Spina V, et al. Mutations of the SF3B1 splicing factor in chronic lymphocytic leukemia: association with progression and fludarabine-refractoriness. Blood 2011; 118: 6904-8.

7. Jeon GW, Han SW, Jung JM, Kang MS, Sin JB. The first Korean case of cutaneous lung tissue heterotopia. J Korean Med Sci 2010; 25: $1387-9$. 